\title{
Reliable Flooding in Dense Backscatter-based Tag-to-Tag Networks
}

\author{
Dilushi Piumwardane, Christian Rohner, Thiemo Voigt ${ }^{1}$ \\ Uppsala University, Sweden , ${ }^{1}$ Uppsala University and RISE Computer Science, Sweden \\ dilushi.piumwardane@it.uu.se, christian.rohner@it.uu.se, thiemo.voigt@it.uu.se
}

\begin{abstract}
RFID and backscatter allow for extremely lowpower or battery-free tags by outsourcing the generation of the radio carrier wave to an external device such as an RFID reader. Recent advances in backscatter communication enables tags to both transmit and receive standards-compliant packets with submilliwatt power consumption. The ability to receive packets makes multi-hop tag-to-tag networking possible, a task that current backscatter networks provide only for limited topologies. Tag-to-tag networks further allow for novel applications such as wireless robotic materials that inherently require dense networks of such tags. In contrast to conventional networks, the tags' communication range in such networks depends heavily on the signal strength of the carrier wave at the transmitter and the receiver. In this paper, we demonstrate for the first time on real hardware multi-hop in backscatter-based networks using standards-based protocols. We present analytical and simulation results that show that both the output power and the position of the carrier generator impact the reliability of the network. We finally demonstrate that simple flooding with a random forwarding delay is an efficient solution for transfering data in such networks.
\end{abstract}

\section{INTRODUCTION}

RFID and backscatter enable extremely low-power or battery-free tags by outsourcing the generation of the radio carrier wave to an external device such as an RFID reader. Backscatter-based tag-to-tag networks, where tags can communicate with each other, have recently been identified as an attractive and open problem in backscatter networking [15]. Enabling multi-hop backscatter-based tag-to-tag networks, that interoperate with standard protocols (Figure 1), opens new opportunities. The battery-free tags can be placed in hard to reach places, such as under the ground, in bodies, thick walls and different materials. Dense tag-to-tag networks enable novel applications such as wireless robotic materials [3] that consist of a large number of sensors and actuators. Such applications require communication for triggering actuation tasks on sensor events. In case the sensor cannot directly reach the actuator, multi-hop communication is required. We have recently proposed backscatter-based communications for robotic materials [11].

Recent progress in backscatter includes systems that interoperate with standard protocols such as ZigBee (IEEE 802.15.4) and WiFi [8]. Moreover, extremely low-power receiver architectures that are able to receive standards-based packets since they, in contrast to envelop detectors, can handle phase and frequency have been developed [4], [13]. While there have been recent attempts to introduce tag-totag networks [9], [17], [18], these attempts have been limited

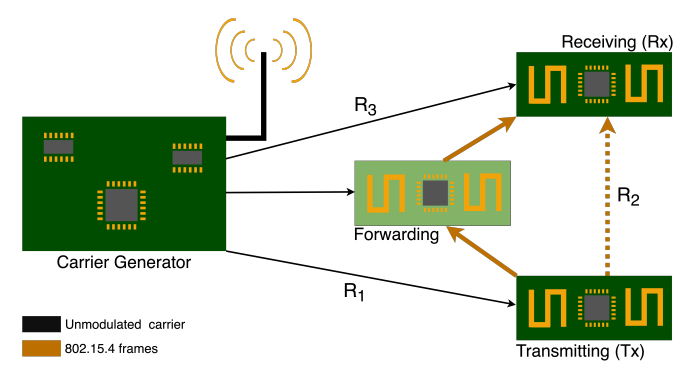

Fig. 1: Standards-compliant tag-to-tag communication. The communication range depends on the distances $R_{1}, R_{2}, R_{3}$ for fixed carrier generator output power. If $\mathrm{Rx}$ is not within the range of $\mathrm{Tx}$, forwarding nodes can relay.

in hops and network layouts. In this paper, we demonstrate backscatter-based tag-to-tag networks that rely on such standards-based receiver architectures. We also explore how to efficiently communicate in such networks with a focus on dense networks.

Contributions. After presenting some background knowledge, we discuss in Section III how our backscatter-based networks differ from conventional wireless networks in terms of communication range.

Section IV discusses specific characteristics of backscatterbased multi-hop communication. These are important when a sensor node needs to transfer data, for example, an actuation command, to an actuator and the actuator is not in the communication range of the sensor. While we can increase the strength of the incident carrier wave and hence the output power of the carrier generator, this is not always desirable. A higher output power increases network interference, power consumption of the carrier generator and may violate safety requirements for sensitive applications. We show that reducing the output power of the carrier generator in such networks decreases the number of paths in the network which may impact delay and reliability. Furthermore, our study reveals that not only the carrier's output power but also its position has a strong impact on the performance of the network.

Finally, in Section V we show with experiments on real hardware that decreasing the output power of the carrier generator is not necessarily detrimental to the reliability in dense networks. This is the first experiment that demonstrates a tag-to-tag backscatter network supporting the IEEE 802.15.4 standard on real hardware. In addition, we conduct simulation experiments for standards-compatible carrier-assisted 
communications using the extensions of Cooja simulator [12]. They indicate that with a random forwarding delay, simple flooding is efficient to transport data from a source node to a target node, over multiple hops in dense backscatterbased networks. Such a simple mechanism is viable also for resource-constrained backscatter nodes.

To summarize, we make the following contributions in this paper:

- We demonstrate for the first time on real hardware multi-hop in backscatter-based sensor networks using standards-based protocols.

- Our analytical and simulation results show that both the output power and the position of the carrier generator impacts the reliability of the network.

- We demonstrate the efficiency of simple flooding with a random forwarding delay in dense backscatter-based networks.

\section{BACKSCATTER COMMUNICATIONS}

In this section we introduce backscatter communications, pointing out important differences to conventional radios. For transmission, the devices use backscatter communications, while for reception of standards-based packets (ZigBee, Bluetooth etc.) they typically employ receivers that in order to reduce power consumption use an external carrier instead of a local oscillator [4], [13]. We present the operating principles of each of these techniques and the resulting signal strength needed for communication.

Backscatter transmissions. Backscatter transmitters work by reflecting an external RF signal to convey useful information [8]. This technique allows transmitting data with up to three orders of magnitude lower power consumption than traditional radios, as transmitters do not need to generate their own RF waves. An important technique that enables backscattering standard commodity wireless protocols is to reflect the signal at a different frequency than the necessary unmodulated carrier. This allows the receiver to avoid interference from the strong carrier signal.

Carrier-assisted receiver. An external carrier can help a receiver operate with a power consumption well under $1 \mathrm{~mW}$ [4], [13] while remaining compatible with unmodified commodity devices. Such a receiver sidesteps power-hungry blocks commonly found in traditional radio receivers such as local oscillators and Analog-to-Digital Converters (ADCs) by employing passive circuits. Specifically, it offloads the local oscillator (LO) to an external device that broadcasts an unmodulated carrier. The receiver also has a passive diode mixer to downconvert the RF signal to a low Intermediate Frequency, where it can be treated easily and efficiently.

Signal strength for backscatter transmissions. The Radar Range Equation [1], [8] describes the power $\left(P_{r}\right)$ of a backscattered signal observed at a receiver and coming from a backscatter device separated from the carrier generator by a distance $R_{1}$ and from the receiver by a distance $R_{2}$ as shown in Figure 1.

$$
P_{r}=\left(\frac{\lambda^{2} P_{t} G_{t}}{16 \pi^{2} R_{1}^{2}}\right)\left(G_{b}^{2} \alpha \frac{|\Delta \Gamma|^{2}}{4}\right)\left(\frac{\lambda^{2} G_{r}}{16 \pi^{2} R_{2}^{2}}\right)
$$

Here $P_{t}$ is the output power of the unmodulated carrier, $G_{t}, G_{b}$ and $G_{r}$ are the antenna gains of the carrier generator, battery-free device and receiver respectively, $\lambda$ is the wavelength of the signal, $\alpha$ is a constant that describes losses incurred in modulating the signal using backscatter and $|\Delta \Gamma|^{2}$ is the backscatter coefficient [8], which is a measure of the efficiency of the backscatter process.

Perez-Penichet [13] devised the following empirical model for the sensitivity threshold $S_{t h}$ based on a real carrier-assisted prototype:

$$
S_{t h}=\frac{C}{P_{i}}
$$

Here $P_{i}$ is the incident carrier power and $C=10^{-12.5}$ is a constant obtained experimentally.

To model how $S_{t h}$ varies with the carrier output power we employ the Friis Equation [1] to compute $P_{i}$ :

$$
P_{i}=P_{t} G_{t} G_{r} \frac{\lambda^{2}}{16 \pi^{2} R_{3}^{2}}
$$

Where $R_{3}$ is the distance between the receiver and the carrier generator. We base our analysis on the assumption that the signal power at the receiver $P_{r}$ must overcome the sensitivity threshold $S_{t h}$ of the receiver for successful decoding of the data. This leads to the condition: $P_{r}>S_{t h}$. Therefore,

$$
P_{t}>\frac{\sqrt{C}}{G_{t} G_{b} G_{r} \sqrt{\alpha} \frac{|\Delta \Gamma|}{2}} \frac{4 \pi R_{1}}{\lambda} \frac{4 \pi R_{2}}{\lambda} \frac{4 \pi R_{3}}{\lambda}
$$

In Equation 4, the minimum power needed from the carrier generator depends on three variables: $R_{1}, R_{2}$ and $R_{3}$. The rest of the equation is a constant for a given backscatter tag.

\section{COMmunicATION RANGES}

In this section, we discuss how the communication range in backscatter-based tag-to-tag networks is affected by the external carrier using analytical results based on the principles and equations obtained in Section II.

Transmission Range Depends on Strength of Carrier Wave. In conventional wireless sensor networks the transmission range is determined by the transmit power of the sender's radio. Backscatter tags cannot control the transmit power, as they work by reflecting an external RF signal such as a carrier wave. The transmission range is thus dictated by the strength of the impinging carrier wave which is determined by the location and output power $P_{t}$ of the carrier generator. This implies that the nodes in a backscatter-based network have different transmission ranges dependent on their distance from the carrier generator. The further away a tag is from the carrier generator, the shorter is its transmission range.

Sensitivity Threshold. While conventional RF receivers have a constant sensitivity threshold that depends on the receiver design and the used modulation, our battery-free receiver, depend on the impinging carrier wave as well. As established in Section II, a node's sensitivity threshold increases (i.e., the receiver is less sensitive) as the strength of the carrier wave 


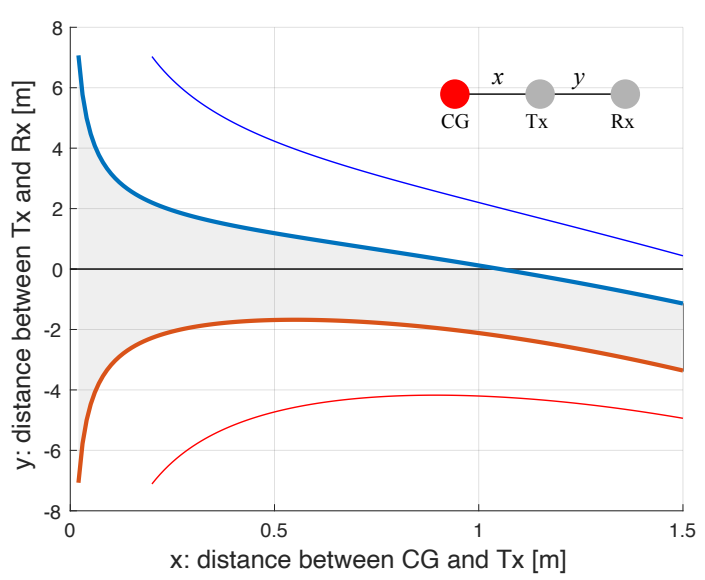

Fig. 2: Communication range (range between the red and blue line) in tag depends on its distance to carrier generator. The fine blue and red lines correspond to a carrier generator with 10 times the output power $P_{t}$.

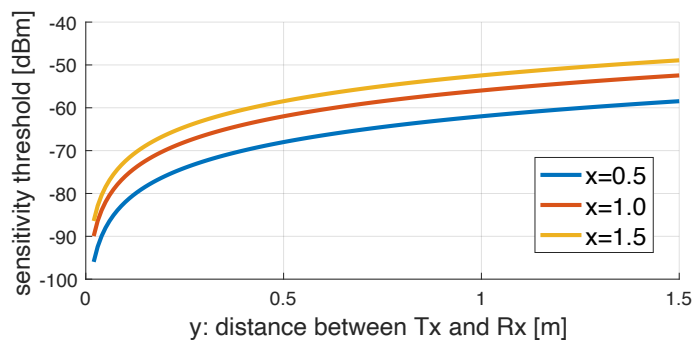

Fig. 3: The sensitivity of the tag receiver depends on the incident carrier power. The sensitivity threshold increases with the distance to the carrier generator and the transmitter increases, making the tag less sensitive for reception.

is getting weaker. Successful reception of a signal depends on the received signal strength from both the transmitter as well as the carrier generator.

The Resulting Communication Range. To illustrate the overall consequences of the dependency of aspects from transmission and reception, we calculate the communication range on a simple scenario where carrier generator, transmitter tag and receiver tag are located in line with distances $x$ and $y$ in between, respectively. We assume $P_{r}=S_{t h}$ to be the boundary of the communication range $y$ when the received signal $P_{r}$ is equal to the receiver sensitivity $S_{t h}$. The inequality of Equation 4 can thus be written as equality and for the purpose of establishing an intuition be simplified to $P_{t}=\beta R_{1} R_{2} R_{3}$, or $P_{t}=\beta x y(x+y)$ in our in-line scenario. Solving this quadratic equation in $y$ yields the following two results, corresponding to the boundary of the communication range away from the carrier generator, and one towards the carrier generator.

$$
y=-\beta x^{2} \pm \frac{\sqrt{\beta^{2} x^{4}+4 \beta x P_{t}}}{2 \beta x}
$$

The solution for the communication range $y$ is illustrated in Figure 2, assuming $\beta=1$ and $P_{t}=1$. Besides the wellknown decrease of the communication range with increasing distance to the carrier generator, the takeaway message from this figure is the asymmetric behavior of the communication range in the direction away from the carrier generator and transmitting tag compared to the opposite direction. Beyond a certain distance $x$ between the carrier generator and the transmitting tag, the communication range does not cover any forward-directed reception (in the given scenario for $x>1$ ). This range can be extended by increasing the output power of the carrier generator, as illustrated with the thin lines corresponding to $P_{t}=10$. Figure 3 shows how the sensitivity threshold increases (i.e., the receiver becomes less sensitive) when the distance $y$ between the transmitting and the receiving tag increases for different distances $x$ between the carrier generator and the transmitting tag. The figure shows a strong increase in the sensitivity threshold for all distances. This is in sharp contrast to conventional receivers in low-power wireless networks where the sensitivity threshold is constant for a given node (even though it typically varies between different nodes of the same model).

\section{MULTI-HOP CHARACTERISTICS}

Dense network applications like wireless robotic materials consist of both sensor and actuator nodes. Certain sensor events have to trigger actions by one or more actuators. Hence the sensing events need to be communicated from a sensor to an actuator. In case the sensor cannot directly reach the actuator, multi-hop communication is required where a tag forwards a received message on behalf of the original sensor. While with sufficiently strong carrier waves, single-hop could be achieved, we may want to reduce the output power of the carrier generator for several reasons: (i) to reduce interference, both intra-network and external interference; (ii) we may want to reduce the energy consumption of the carrier generator in case it is battery-powered; (iii) application constraints such as safety requirements for on- or in-body networks may not allow high output power. In this section, we study the characteristics of backscatter-based multi-hop communication. The goal is to understand the basic characteristics of this new communication paradigm and how we can exploit them to achieve reliable communication under energy constraints.

\section{A. Basic properties of Multi-Hop Communication}

As discussed in Section III, the communication range of tags depends on the strength of the impinging carrier signal. The incident power of the carrier signal on the tag depends on the location and output power of the carrier generator. In this section, we first study analytically the impact of the location (in terms of distance and angle) of the carrier generator on the multi-hop performance.

Figure 4 shows the setup for this analysis. The setup consists of three tags and one carrier generator, forming the simplest multihop tag-to-tag network. The tags are distributed uniformly with equal distance between them. The distance between the carrier generator and Tag 2 is $c$ and the angle is $\varphi$. We set the distance between Tag 1 and Tag 3 to 1 . The distances $R_{1}$ and $R_{3}$ are calculated using basic trigonometric formulas. In the figure, a single hop is from Tag 1 to Tag 3 while multi-hop consists of two hops from Tag 1 to Tag 2 
and from Tag 2 to Tag 3. To form a linear network, we set the angle $\varphi$ to 0 to compute the minimum power required to transfer a packet from Tag 1 to Tag 3 (either in a single or two hops) as a function of the distance. We then set the distance $c$ at 0.75 to compute the relative minimum power as a function of the angle. The plots in Figure 5 show the change in the minimum carrier power when varying angles and radius when the carrier generator moves along the circle.

Impact of the distance of carrier generator. In Figure 5a, when $\varphi=0$, the tags and the carrier generator are in line. The graph shows the minimum power consumption for the single hops with thinner lines, i.e., links from Tag 1 to Tag 2 (link 1,2), Tag 2 to Tag3 (link 2,3) and from Tag 1 to Tag 3 (link 1,3). It also shows the minimum power for the paths with thicker lines: the multi-hop path from Tag 1 to Tag 3 via Tag 2 (path 1,2,3) and the direct path from Tag 1 to Tag 3 (path 1,3). The latter is on top of link 1,3, so the yellow link is never visible in the graph.

For the distance $c=0.5$ the carrier generator's location overlaps with the receiver's location. Hence, according to Equation 4 the power consumption is zero as $R_{3}$ is zero when Tag 3 is the receiver. The power consumption is 0 is caused by our modeling assumptions.

The minimum power consumption of the carrier generator for the multi-hop path $1,2,3$ is always the maximum link power consumption of link 1,2 and link 2,3, i.e., path $1,2,3=\max ($ link 1,2 ; link 2,3$)$. When $\varphi=0$, this is always link 1,2 since it is further away from the carrier generator than link 2,3 , hence path $1,2,3=\operatorname{link} 1,2$ and the thin blue line is never visible in the graph.

For smaller distances $(c<1)$, the path 1,3 has lower power consumption than path $1,2,3$. The reason for this is that in this case, the carrier generator is quite close to Tag 3 and hence Tag 3's sensitivity threshold is lower (favourable) compared to the one of Tag 2 that is the receiver for the link 1,2, the first hop of path 1,2,3. For distances larger than $c=1$, however, multi-hop (path 1,2,3) requires less carrier generator power than single-hop (path 1,3). Here, the power consumption is dominated by the energy for bridging the distances rather than the sensitivity threshold.

Impact of the angle of carrier generator. Figure $5 b$ shows the minimum carrier output power needed when the carrier generator moves along the circle depicted in Figure 4, for a distance of $c=0.75$. At this distance and with $\varphi=0$, path 1,3 requires less carrier output power than path 1,2,3 as shown in Figure 5a. While the carrier generator is moving towards $\varphi=0.5$, its distance to Tag 3 increases which increases the minimum power required for path 1,3 . On the other hand, the minimum carrier power for path 1,2,3 decreases when the carrier generator moves towards $\varphi=0.5$, since the distance R1 decreases and hence the minimum carrier power for link 1,2 decreases. The situation is roughly symmetric for angles between $\varphi=0.5$ and $\varphi=1$, with the difference that now link 2,3 dominates path 1,2,3's required minimum carrier power rather than link 1,2 since link 2,3 is now further from the carrier generator than link 1,2.

Both figures contain areas where multi-hop reduces the

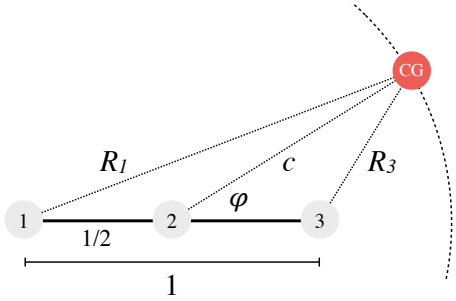

Fig. 4: Carrier generator location impact for multihop Angle $(\varphi)$ and radius $(c)$ with the middle tag (2) is used as a reference to calculate carrier generator position to other tags. Tags are uniformly distributed.

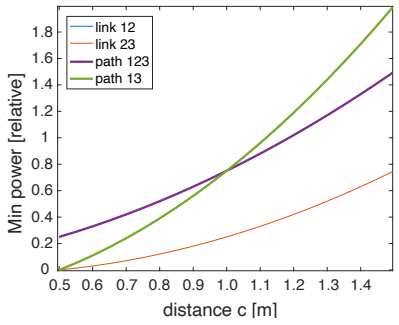

(a) Distance $c$ when angle $\varphi=0$

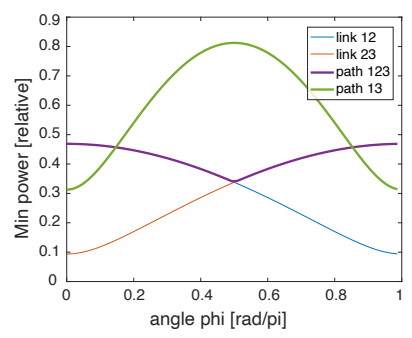

(b) Angle $\varphi$ when distance $c=$ 0.75
Fig. 5: The location and angle of the carrier generator contribute to the minimum requried carrier power.

required carrier generator output power compared to a larger single hop. While multi-hop adds some additional forwarding delay, it has the benefit of requiring less strong carrier waves which causes less external interference.

\section{B. Least-cost Multi-hop Paths}

When a packet is transmitted from a sensor to an actuator, it generally travels on multiple links, forwarded by a number of nodes. As shown above, the power required for a link depends on the strength of the incident carrier wave and hence the location and output power of the carrier generator. The link of the path that requires the highest incident power determines the minimum output power of the carrier generator that is required to transport a packet across the path. The goal of this analytical experiment is to understand the impact of the location of the carrier generator on the power required to transmit a packet on the least cost multi-hop path.

Setup. We place the carrier generator in nine different locations in a circle as in shown Figure 6a. Nodes 1 and 6 are source and destination, resp. In this scenario, there are three different paths from source to destination, each with four nodes including source and target. To determine the least-cost path we use a modified version of the Dijkstra algorithm.

Result. Figure $6 \mathrm{~b}$ shows the result. The minimum power required for the least cost path changes as the location of the carrier generator changes. This is because the relative position to the carrier generator for different links in a given path changes, which affects the cost of the path. We take the link with maximum cost as the cost of the path, and the link and its cost depend on the carrier generator location which controls the incident power on tags. This is different from 


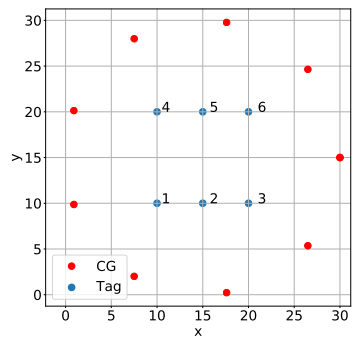

(a) Layout of Carrier Generator (Red) and Tags (Blue).

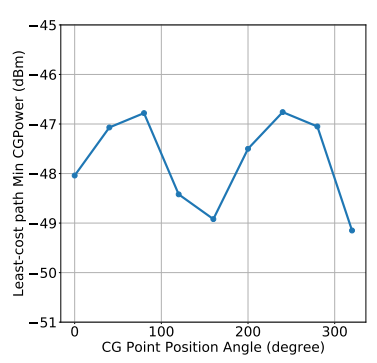

(b) Power required for the leastcost path
Fig. 6: Power required for the least-cost path. For each location of the carrier generator, the minimum power consuming path is calucated from source (1) to target (6). This path is chosen from multiple paths from source to target, according to the link with the highest power consumption.

conventional IoT networks as the performance of nodes in the network depends on an external device.

\section{Number of Multi-hop Paths}

The number of paths in a wireless multi-hop network determines the overall number of packet transmissions when a message is sent from a source to a sink. Therefore, it has an impact on both the delay and the reliability. We analyse the number of paths for a single source $(\mathrm{Rx})$ that sends a single message to a single destination (Tx) in the network. As discussed in Section III, the incident power on tags determines their transmission range and hence the number of potential receivers and paths. The goal of this analytical experiment is to study the impact of the carrier position and strength on the number of paths between a given source and target.

Setup. Figure 7 (left) shows the layout of a $5 \times 5$ grid and three different carrier positions with the same distance from the center of the grid. Position 1 is close to $T x$, resulting in a large communication range for $T x$. Position 3 is close to $R x$, resulting in higher sensitivity for nodes close to Rx. Finally, position 2 is chosen to be equally distant from $R x$ and $T x$. We vary the carrier strength and count the number of possible paths. We restrict every node to forward a message only once.

Result. Figure 7 (right) shows the result. The black dashed line indicates the power needed for Position 1 and 3 to support one-hop communication between $\mathrm{Tx}$ and $\mathrm{Rx}$.

When the carrier is closer to $T x$ (pos 1), a large number of nodes receive the message directly from $T x$ and forward it. $R x$, however, due to its reduced sensitivity, only receives from its closest neighbors and the number of paths is therefore limited and bounded to at most 24 paths for a strong carrier; one one-hop path and 23 two-hop paths.

When the carrier is closer to $R x$ (pos 3), the transmission from $T x$ only reaches its closest neighbors due to the distance from the carrier. Subsequent forwarding transmissions reach increasingly longer, leading to a large diversity of arising paths. As the carrier is strong enough for $T x$ to reach every node, the number of paths drops to 24 as for position 1 .

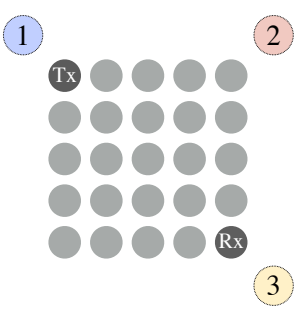

(a) Experimental Setup

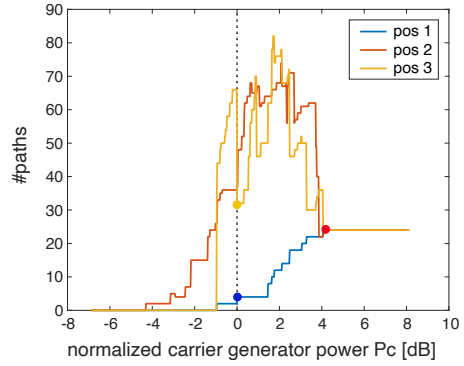

(b) No. of Possible Paths
Fig. 7: Paths from source (Tx) to target $(\mathbf{R x})$ as carrier power increases for different carrier generator positions. The power is normalized to the minimal power required for a one-hop path in positions 1 and $3(0 \mathrm{~dB})$.

When the carrier is at an equal distance from $T x$ and $R x$ (pos 2) these two effects play together, leading to a rich set of paths for medium carrier strength. Interestingly, this scenario is the one allowing end-to-end paths with lower carrier power than the other two scenarios. The two least-cost paths pass through the upper right part of the network and have 4 and 5 hops, respectively.

\section{TOWARds REliable Flooding}

In this section, we present results for tag-to-tag networks from both simulations and experiments on real hardware (Figure 9). As the nodes in our networks are extremely resourceconstrained we investigate whether flooding-based solutions are sufficiently reliable in the dense networks we target.

\section{A. Impact of Carrier Power on Packet Reception Rate}

Our experiments in the previous sections have shown that reducing the output power of the carrier generator reduces the number of paths from a sensor (source) to a sink. The experiment in this section is done on real hardware to investigate whether in a setting with real backscatter-nodes such a reduction of the output power of the carrier generator and hence the communication range of the nodes is viable, i.e., the low output power is not detrimental to reliability.

Setup. The experimental setup is depicted in the left part of Figure 9. A USRP B200 device provides the unmodulated carrier necessary for the tags to backscatter and receive data. It generates a carrier at $2.472 \mathrm{GHz}$. We vary the gain of the USRP and hence the output power of the carrier generator. To obtain higher transmit powers (from $17 \mathrm{dBm}$ onwards) an external amplifier is connected to the USRP device. Each of the four FPGA-based tag prototypes consists of a transmitter and a receiver that can send and receive IEEE 802.15.4 packets [13]. The transmitter tag shifts the carrier frequency $8 \mathrm{MHz}$ to avoid strong interference from the carrier at the receiver. We place the carrier generator $65 \mathrm{~cm}$ from the transmitter tag. We put the receiver tags at approximately the same distance from the transmitter tag as shown in Figure 8a. The transmitter tag periodically sends standards-compliant 802.15 .4 packets with a sequence number that the receiver tags record. We send 1200 


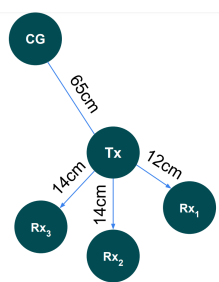

(a) Experimental Setup

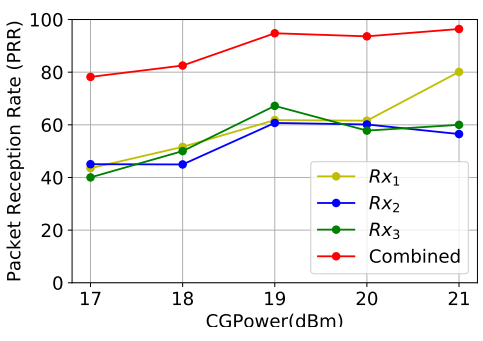

(b) Individual and Combined PRR
Fig. 8: PRR of tags increase as the carrier-generator (CG) output power increases. The combined PRR is high, which is beneficial for dense networks.

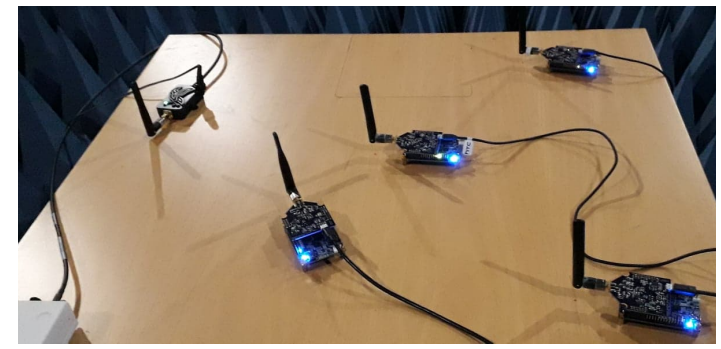

Fig. 9: Example experimental setup of a tag-to-tag network.

packets for each output power. We perform the experiment in an indoor office environment, where external interference is present.

Result. Figure 10b shows the results. The individual tags' packet reception rate (PRR) increases with the carrier generator's output power. While the individual links have a low PRR when the carrier's output power is low, their combined PRR is nevertheless high. We take the union of PRR of individual tags, as the combined PRR. This implies that in a dense network, a flood can still continue without compromising the reliability of the network, despite losses on individual links.

\section{B. Impact of Multi-hop on Packet Reception Rate}

After observing individual links in the previous experiment, we conduct this experiment to observe if multi-hop is effective to increase PRR in our tag-to-tag networks. When the carrier generator is placed close to a tag, it has better reception and transmission ability than the tags placed further away (Section II). In a tag-to-tag network, when an initiating transmitter tag wants to send data to a receiver, the communication range is affected by the relative placement to the carrier generator. In a sufficiently dense network, there are tags placed relatively closer to the carrier generator that can be used to forward the messages from the transmitter to the target tag. This experiment evaluates such a scenario.

Setup. Tag $T_{x}$ periodically broadcasts 350 packets with sequence numbers. Tag $N_{1}$ adds a unique ID and broadcasts the packets it receives, after a constant delay, which is less than the broadcast period of tag $T x . R_{x} \operatorname{logs}$ all packets that are received from $T_{x}$ and $N_{1}$. The carrier generator is placed closer to the forwarding node. We then extend this experiment to measure PRR, by using tag $N_{2}$ also to forward packets. As

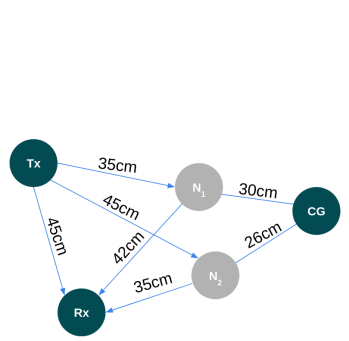

(a) Experimental Setup

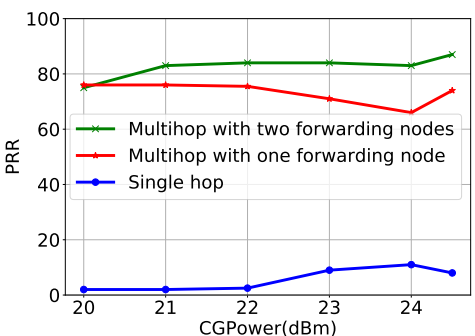

(b) PRR of nodes when using single hop and multihop.

Fig. 10: PRR of $R_{x}$ receiving through $N_{1}$ (red) significantly improves compared to $R_{x}$ directly receiving from $T_{x}$ (blue). PRR is higher when receiving through both $N_{1}$ and $N_{2}$ (green). $T_{x}$ sends packets with sequence numbers and the received packets are counted as percentage of the original sequence numbers.

in the previous experiment, we vary the output power of the carrier generator.

Result. Figure 10 shows that tag $R_{x}$ is able to receive more packets via $N_{1}$ than it can directly receive from $T_{x}$. This is because tag $N_{1}$ receives more packets than tag $R_{x}$, as the carrier generator is closer to it than to $R_{x}$, which improves the sensitivity of $N_{1}$. Tag $N_{1}$ also has more transmit power than the tag $T_{x}$ as it is closer to the carrier generator. When tag $N_{2}$ also contributes to forwarding packets, the PRR of tag $R_{x}$ increases further. Even though the distance between $T_{x}$ and $N_{2}$ as well as $T_{x}$ and $R_{x}$ is the same, because of $N_{2}$ 's position, it can receive and transmit better. This behaviour is different from regular IoT devices where if $N_{2}$ is in the communication range of $T_{x}$, the same is true for $R_{x}$. Note an increase of the CG power has only a moderate impact on the PRR for the single hop from $T_{x}$ to $R_{x}$.

This result shows that forwarding by relay nodes placed in positions where sensitivity or transmit power is higher, helps to improve the PRR at the target node. In dense networks, multiple relay nodes can act collectively to forward packets and increase the PRR.

\section{Multi-hop in Dense Networks}

In a larger tag-to-tag network, for a given position of the carrier generator, there can be many nodes on the paths from $T_{x}$ to $R_{x}$ that contribute by forwarding packets. Understanding these forwarding paths can help increase the reliability and reduce interference and traffic in the network. We conduct experiments in larger multi-hop tag-to-tag networks with simulations.

Setup. We simulate our networks in the COOJA simulator with extensions that support standard-compatible carrier-assisted communications [12]. The network consists of $8 \times 8$ grid and one carrier generator. We place the carrier generator in three different positions similar to those in Section IV-C, with equal distance to the center of the network as shown in Figure 11a. Position 1 and Position 3 provide just enough power for a packet to reach $R_{x}$ from $T x$ in one hop.

The carrier generator emits an unmodulated carrier (radio test mode) on channel 18 at $0 \mathrm{dBm}$. The tags receive at 
(1)

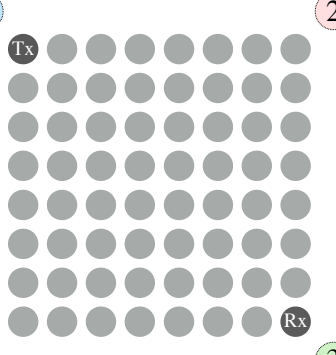

3

(a) Tag-to-tag Network Setup

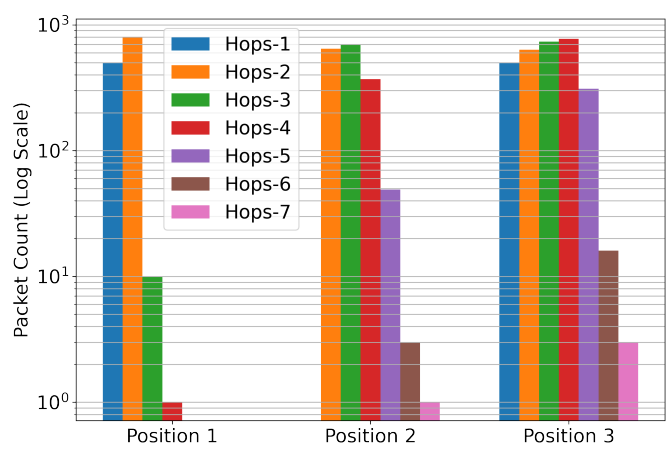

(b) Distribution of No. of Hops

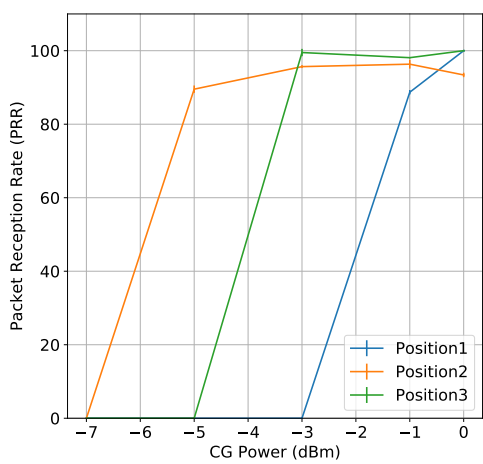

(c) Packet Reception Rate

Fig. 11: No. of Hops used to forward packets from $T_{x}$ to $R_{x}$. Number of hops for copies of the packet are different even if $R_{x}$ is directly receiving from $T_{x}$ with identical $P_{t}$ values. Therefore the paths taken to forward the packet are different even if the distance to the carrier generator from the network is the same. PRR drops when carrier power is too high or too low.

channel 20 to account for the frequency-shifted backscatter at the transmitter tag. When forwarding nodes receive a packet, they re-broadcast the packet after a maximum random delay of $15 \mathrm{~ms}$. Packets can be lost due to collisions and interference with other packets or when there is no receiver in the communication range. In a second step, we extend the experiment with varying carrier power and measure the PRR at positions 1,2 and 3 . For each position, we send 500 packets from $\mathrm{Tx}$ and repeat this for three different random seeds.

Results. Figure $11 \mathrm{~b}$ characterizes the paths through the network by their number of hops. When the carrier is in Position 1 , since all nodes receive from $T_{x}$, they are all scheduled within the first $15 \mathrm{~ms}$ to forward the packet. Therefore the chances for collisions are high and the number of successful paths is low. When the carrier generator is in Position 3, all nodes do not receive from $T_{x}$, and therefore the ones that receive (1st hop), schedule the packet forwarding within $15 \mathrm{~ms}$. Since $R_{x}$ can receive from all nodes in this carrier generator position, if there are no collisions, $R_{x}$ can receive through many different paths. For a given packet, this may lead to more copies of the packet distributed in a larger time frame at the receiver, with many hops. When the carrier is placed in Position 2, $R_{x}$ receives packets for lower carrier powers, than when it is in Position 1 and 3, as discussed in Section IV.

Figure 11c shows the packet reception rate for the three carrier positions for different carrier powers. First, we notice that different carrier strengths are required for successful reception at the three carrier positions. Second, the best reception is not achieved with the highest carrier power. This can be explained by looking at where collisions are likely to happen. For a low carrier power, $R_{x}$ receives only from a few neighbor nodes which leads to a lower reception rate. If the packet cannot reach those nodes, due to collisions in earlier hops, the packet cannot reach $R_{x}$. When the carrier power is high, many nodes receive packets from $T_{x}$ in the first hop. They all schedule the next transmission within $15 \mathrm{~ms}$, which leads to a higher number of collisions. The interference at $R_{x}$ is also high since it can receive and be interfered from many nodes when the carrier

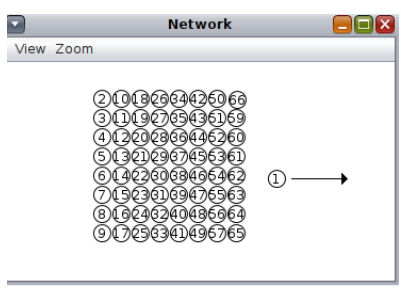

(a) Tag-to-tag Network Setup

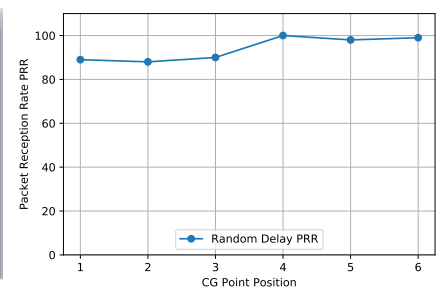

(b) Packet Reception Rate
Fig. 12: PRR for random delay of packet forwarding. Nodes broadcast the packets with a random delay. Some packets are lost when carrier generator is close due to interference and when it is far due to few links to reach target.

power is high.

\section{Is Simple Flooding Reliable?}

The goal of this experiment is to study if simple flooding is reliable in backscatter-based multi-hop networks.

Setup. We borrow the experimental setup for COOJA, from the previous experiment. We place the carrier generator linearly with the center of the tag at six different locations with increasing distances from the network. A packet needs to be forwarded from a source node to a target node as shown in Figure 12a. Nodes forward a packet by broadcasting it so that the neighbors within the communication range receive and forward it. We explore two different options: (i) a simple flooding option where nodes forward a received packet within a constant delay and (ii) nodes forward a packet within a random delay of up to $20 \mathrm{~ms}$.

Results. When the forwarding delay of the nodes is constant, the target does not receive any packets. The reasons for this are collisions that typically happen within a few hops from the sink.

In contrast, Figure 12 shows that with the random delay only a few packets are lost. The results also indicate that more packets are lost when the carrier generator is close to the network (Positions 1 and 2). As discussed in the previous 
section this increases the number of paths in the network which may lead to high intra-network interference, due to high interference which causes collisions and packet losses. When the carrier generator is farther away (Positions 5 and 6 ), the target can only be reached by a few closer nodes since the communication range is reduced. When packets do not reach those few nodes due to collisions in previous hops, no packets are forwarded to the target. This implies that the network is not dense enough for reliable flooding. At Position 4, all packets are received when the carrier generator is far away from the network to cause lower communication ranges while the network is still dense enough to enable reliable flooding. In summary, our results suggest that a simple flooding mechanism with random forwarding delay is efficient for tag-to-tag networks.

\section{RELATED WORK}

Similar to RFID, early studies in backscatter and computational RFID have focused on single-hop networks using platforms such as Moo [16] and WISP [2], [14]. More recent efforts have introduced multi-hop backscatter networks [9], [17], [18] in form of chain networks with only a single path from transmitter to receiver. Their networks have typically employed envelop detectors as receivers. As Galisteo et al. point out such envelop detectors have limited sensitivity and are affected by external interference and ambient traffic [6]. Moreover, they are not able to receive standards-based Internet of Things packets from e.g. ZigBee, Bluetooth WiFi transmitters since they focus on the amplitude of the signals rather than phase and frequency.

In contrast to these networks, we aim at dense tag-to-tag networks rather than chain networks based on standard IoT protocols. Such networks require receivers that can decode standards-based protocols at very low power consumption. Recent backscatter devices support such standard-based receptions [4], [13]. One of the main features of their receivers is that they replace the energy-hungry local oscillator with an external carrier wave. This implies, however, that the sensitivity threshold of the receiver is not constant as in conventional radios but depends on the strength of the carrier wave. As we discuss in this paper, therefore the placement and strength of the carrier generator have a strong impact on the performance of the tag-to-tag networks. As Katanbaf et al. discuss, the sensitivity threshold also needs to be considered in bistatic backscatter systems [7] even though its impact is more profound in tag-to-tag backscatter networks.

Our experiments suggest that in dense tag-to-tag backscatter-based networks, simple flooding is efficient. Glossy [5] is an extremely efficient flooding solution for sensor networks but does not work with backscatterbased networks since they do not support constructive interference [10].

\section{CONCLUSIONS}

In this paper we have studied the properties and performance of standards-compliant backscatter-based tag-to-tag networks, which enable novel applications such as wireless robotic materials. As nodes are extremely resource-constrained and the networks we target dense, we investigate whether simple flooding-based solutions are efficient in these networks. Our results indicate that flooding with a random forwarding delay is a viable solution in dense backscatter-based networks when sending a packet from a source to a sink.

\section{Acknowledgements}

We thank our sheperd Cheng Qi. This work has been supported by the Swedish Research Council (Grant 2017045989 and 2018-05480) and the Swedish Foundation for Strategic Research.

\section{REFERENCES}

[1] C. A. Balanis. Antenna Theory: Analysis and Design, 3rd Edition. Wiley-Interscience, Hoboken, NJ, 3 edition edition, Apr. 2005.

[2] M. Buettner, B. Greenstein, and D. Wetherall. Dewdrop: an energyaware runtime for computational rfid. In Proc. USENIX NSDI, 2011.

[3] N. Correll, P. Dutta, R. Han, and K. Pister. Wireless robotic materials. In Proceedings of the 15th ACM Conference on Embedded Network Sensor Systems, SenSys '17, New York, NY, USA, 2017. ACM.

[4] J. F. Ensworth, A. T. Hoang, and M. S. Reynolds. A low power $2.4 \mathrm{GHz}$ superheterodyne receiver architecture with external LO for wirelessly powered backscatter tags and sensors. In 2017 IEEE International Conference on RFID (RFID), pages 149-154, May 2017.

[5] F. Ferrari, M. Zimmerling, L. Thiele, and O. Saukh. Efficient network flooding and time synchronization with Glossy. In ACM/IEEE IPSN, Chicago, IL, USA, April 2011.

[6] A. Galisteo, A. Varshney, and D. Giustiniano. Two to tango: Hybrid light and backscatter networks for next billion devices. In MobiSys. ACM, 2020.

[7] M. Katanbaf, A. Saffari, and J. R. Smith. Receiver selectivity limits on bistatic backscatter range. In 2020 IEEE International Conference on RFID (RFID), pages 1-8, 2020.

[8] B. Kellogg, V. Talla, S. Gollakota, and J. R. Smith. Passive Wi-Fi: Bringing Low Power to Wi-Fi Transmissions. NSDI '16, pages 151164, 2016.

[9] A. Y. Majid, M. Jansen, G. O. Delgado, K. S. Yildirim, and P. Pawełłzak. Multi-hop backscatter tag-to-tag networks. In IEEE INFOCOM 2019IEEE Conference on Computer Communications. IEEE, 2019.

[10] C. Pérez-Penichet, F. Hermans, and T. Voigt. On limits of constructive interference in backscatter systems. In 2017 Global Internet of Things Summit (GIoTS), pages 1-5. IEEE, 2017.

[11] D. Piumwardane, C. M. Pérez-Penichet, C. Rohner, and T. Voigt. Backscatter communication for wireless robotic materials. In Proc. 2019 International Conference on Embedded Wireless Systems and Networks, EWSN 2019, Beijing, China, 2019. ACM, 2019.

[12] C. Pérez-Penichet, G. Daglaridis, D. Piumwardane, and V. T. Modelling battery-free communications for the cooja simulator. In International Conference on Embedded Wireless Systems and Networks (EWSN), 2019.

[13] C. Pérez-Penichet, C. Noda, A. Varshney, and T. Voigt. Battery-free 802.15.4 Receiver. In IPSN, Piscataway, NJ, USA, 2018.

[14] J. R. Smith, A. P. Sample, P. S. Powledge, S. Roy, and A. Mamishev. A wirelessly-powered platform for sensing and computation. In International Conference on Ubiquitous Computing. Springer, 2006.

[15] V. Talla, J. Smith, and S. Gollakota. Advances and open problems in backscatter networking. arXiv e-prints, pages arXiv-2011, 2020.

[16] H. Zhang, J. Gummeson, B. Ransford, and K. Fu. Moo: A batteryless computational rfid and sensing platform. University of Massachusetts Computer Science Technical Report UM-CS-2011-020, 2011.

[17] J. Zhao, W. Gong, and J. Liu. X-tandem: Towards multi-hop backscatter communication with commodity wifi. In Proceedings of the 24th Annual International Conference on Mobile Computing and Networking, MobiCom '18, New York, NY, USA, 2018. ACM.

[18] J. Zhao, W. Gong, and J. Liu. Towards scalable backscatter sensor mesh with decodable relay and distributed excitation. In MobiSys. ACM, 2020. 